\title{
A semi-analytical dispersion model from a steady source of short duration
}

\author{
Modelo de dispersão semianalítico para uma fonte de curta duração
}

Davidson Martins Moreira *1

${ }^{1}$ Centro Integrado de Manufatura e Tecnologia, Salvador, Bahia, Brasil

\begin{abstract}
This paper presents a semi-analytical unsteady-state solution to the atmospheric dispersion equation for substances subject to chemical-physical decay and that allows a description of the time evolution of the concentration field emitted from a source during a release of short duration. This solution is suitable for describing critical events relative to accidental release of toxic, flammable or explosive substances. The results of the sensitivity analysis and of validation testing, with a proper parameterization of the vertical profiles of the wind and eddy diffusivities, were evaluated against experimental data found in the literature.
\end{abstract}

Keywords: atmospheric boundary layer, air pollution, dispersion modeling.

\section{Resumo}

O presente trabalho apresenta uma solução semianalítica no estado não-estacionário da equação de dispersão atmosférica para substâncias sujeitas a decaimento químico e físico e que permite uma descrição do tempo evolutivo do campo de concentração emitido por uma fonte durante uma liberação de curta duração. Essa solução permite uma descrição de eventos críticos relacionados à liberação acidental de substâncias explosivas e inflamáveis. Os resultados da análise de sensibilidade e do teste de validação, com parametrização apropriada dos perfis verticais de vento e coeficiente de difusão, foram avaliados com dados experimentais encontrados na literatura.

Palavras-chave: camada limite atmosférica, poluição do ar, modelo de dispersão.

* davidson.moreira@gmail.com

Recebido: 10/04/2014 Aceito: 10/04/2014 


\section{Introduction}

$\mathrm{T}$ he usual interventions to safeguard environment and people against industrial activities often imply tackling problems connected with the release in the atmosphere of toxic, flammable or explosive substances. Moreover, it is necessary to rely on models that do not require excessive computational resources to describe the time evolution of the concentration fields and the duration of exposure to emitted substances. This often depends on the necessity of providing estimates in a short time and in emergency situations. The diffusion models for steady-state or instantaneous emissions (puffs) are frequently inadequate, since they do not include some important parameters, such as the duration of the release or the duration of the exposure..

Atmospheric dispersion of pollutants has attracted attention of researchers in many ways. It adds have focused on the environmental impact and health hazards; others have worked on various modeling aspects such the meteorological conditions, dispersion mechanisms, removal mechanisms, topographical features, etc. Mathematical modeling has been the case of many of these studies. The importance and the need of mathematical modeling are well known in the scientific community. There are various modeling approaches that have been used effectively in the past to deal with air pollution dispersion (Zannetti, 1990; Seinfeld and Pandis, 1998; Arya, 1999), and many of them utilize analytical approaches (Tirabassi, 1989; Lin and Hildemann, 1997; Seinfeld and Pandis, 1998; Tirabassi, 2003; Sharan et al., 2003). Analytical solutions (as opposed to numerical ones) explicitly take into account all the parameters of a problem, so that their influence can be reliably investigated and it easy to obtain the asymptotic behavior of the solution, which is usually difficult to generate through numerical calculations. Moreover, bearing in mind that the errors inherent to mathematical models are due to the modeling of the physical phenomena (Moreira et al., 2004) and numerical errors, it turns out that the analytical solutions, somehow, eliminate the numerical error of the equation solution except for the round-off error. As a consequence, becomes possible to make a more realistic analysis of the error appearing in the mathematical modeling due to the physical phenomena. Exist a vast literature about the numerical approaches, and, for sake of illustration, we cite the works of Tangerman (1978), Sharan et al. (1997), Brebbia (1981), Chock et al. (1996), Huebner et al. (2001) and Rizza et al. (2003).

The mathematical models of pollutant dispersion in the atmosphere represent an important technical instrument, as for the knowledge of the state of the atmosphere, united to monitoring net, as for the environmental management. Both our scientific understanding and technical developments have been greatly increased by the use of empirical, analytical and numerical models (Tangerman, 1978) to predict the air pollution concentration in atmosphere. For this purpose, the advection-diffusion equation has been largely applied in operational atmospheric dispersion models. In principle, from this equation it is possible to obtain the dispersion from a source given appropriate boundary and initial conditions plus knowledge of the mean wind velocity and concentration turbulent fluxes. The simplicity of the $\mathrm{K}$ theory of turbulent diffusion has led to the frequent use of this theory as the mathematical basis for simulating air pollution dispersion. Despite these well-known limits (Pasquill and Smith 1983), the K closure is widely used in several atmospheric conditions, because it describes the diffusive transport in an Eulerian framework, where almost all measurements are Eulerian in character (i.e., they are collected in a framework fixed with respect to the earth). It produces results that agree with experimental data as well as any more complex model, and it is not as computationally expensive as higher-order closures.

In this work, a step forward is taken toward solving the two-dimensional, unsteady-state advection-diffusion equation by the ADMM (Advection-Diffusion Multilayer Method) (Moreira et al., 2006) method depending on the time release. This solution allows a description of the time evolution of the concentration field emitted from a source during a release lasting time and chemical-physical decay. To validate the results obtained, numerical comparison is undertaken with available results in the literature. To reach our objective, we organize the paper as follows: in section 2, we report the solution of the advection-diffusion equation by the ADMM method. In section 3, we show the turbulent parameterizations. In section 4 , are presented numerical simulations and comparison with experimental data, and finally in section 5 , the conclusions.

\section{The mathematical model}

A typical problem with the advection-diffusion equation involves the solutions of problems corresponding to instantaneous and continuous sources of pollution. More precisely, considering a Cartesian coordinate system in which the $x$ direction coincides with that one of the average wind, the time dependent advection-diffusion equation can be written as (Huang, 1979):

$$
\frac{\partial C}{\partial t}+u \frac{\partial C}{\partial x}=\frac{\partial}{\partial x}\left(K_{x} \frac{\partial C}{\partial x}\right)+\frac{\partial}{\partial y}\left(K_{y} \frac{\partial C}{\partial y}\right)+\frac{\partial}{\partial z}\left(K_{z} \frac{\partial C}{\partial z}\right)+S
$$

where $C$ denotes the average concentration, $K_{x}, K_{y}, K_{z}$, are Cartesian components of eddy diffusivity, $u$ is the longitudinal wind speed and $S$ a source/sink term.

The analytical solution of the Eq. (1) can be obtained assuming (Huang, 1979):

$$
C(x, y, z, t)=f(x, y, t) C^{y}(x, z, t)
$$


The function $f(x, y, t)$ can be expressed as:

$$
f(x, y, t)=\frac{1}{\sqrt{2 \pi} \sigma_{y}} \exp \left(-\frac{\left(y-y_{o}\right)^{2}}{2 \sigma_{y}^{2}}\right)
$$
tion:

and $C^{y}(x, z, t)$ is the solution of the following equa-

$$
\frac{\partial C^{y}}{\partial t}+u \frac{\partial C^{y}}{\partial x}=\frac{\partial}{\partial x}\left(K_{x} \frac{\partial C^{y}}{\partial x}\right)+\frac{\partial}{\partial z}\left(K_{z} \frac{\partial C^{y}}{\partial z}\right)-\lambda C^{y}
$$

where $C^{y}$ is the crosswind integrated concentration and $\lambda$ represents a chemical-physical decay coefficient.

The mathematical description of the dispersion problem represented by the Eq. (4) is well posed when it is provided by initial and boundary conditions. Indeed, it is assumed that at the beginning of the pollutant release the dispersion region is not polluted, this means:

$$
C^{y}(x, z, 0)=0 \quad \text { at } \quad t=0
$$

and a source of constant emission rate $Q$ is assumed:

$$
C^{y}(0, z, t)=\frac{Q}{u}\left[\eta(\mathrm{t})-\eta\left(\mathrm{t}-\mathrm{t}_{\mathrm{r}}\right)\right] \delta\left(z-H_{s}\right) \text { at } x=0
$$

where $\delta\left(z-H_{s}\right)$ is the Dirac delta function, $H_{5}$ the source height, $\eta$ is the Heaviside function and $t_{r}$ is the duration of release (Bianconi and Tamponi, 1993).

The pollutants are also subjected to the boundary conditions:

$$
K_{z} \frac{\partial C^{y}}{\partial z}=0 \quad \text { at } \quad z=h
$$

and

$$
K_{z} \frac{\partial C^{y}}{\partial z}=0 \quad \text { at } \quad z=0
$$

where $h$ is the height of the Atmospheric Boundary Layer (ABL).

In the following we assume that $K_{x}, K_{z}$ as well the wind speed $u$ depend only on the variable $z$ and we assume an averaged value. The stepwise approximation is applied in problem (4) by discretization the height $h$ into sub-layers in such manner that inside each sub-layer, average values for $K_{x}, K_{z}$ and $\mathrm{u}$ are taken. At this point, it is important to remark that this procedure transforms the domain of problem (4) into a multilayered-slab in the $z$ direction. Furthermore this approach is quite general in the sense it can be applied when these parameters are an arbitrary continuous function of the $z$ variable.

Indeed, it is now possible to recast problem (4) as a set of advective- diffusion problems with constant parameters, which for a generic sub-layer reads like:

$$
\begin{gathered}
\frac{\partial C_{n}^{y}}{\partial t}+u_{n} \frac{\partial C_{n}^{y}}{\partial x}=K_{x, n} \frac{\partial^{2} C_{n}^{y}}{\partial x^{2}}+K_{z, n} \frac{\partial^{2} C_{n}^{y}}{\partial z^{2}}-\lambda C_{n}^{y} \\
z_{n} \leq z \leq z_{n+1}
\end{gathered}
$$

for $n=1: N L$, where $N L$ denotes the number of sub -layers and $C_{n}^{y}$ the concentration at the $n^{\text {th }}$ sub-layer. Besides which, two boundary conditions are imposed at $z=0$ and $h$ given by equation (7) together with the continuity conditions for the concentration and flux of concentration at the interfaces. Namely:

$$
\begin{gathered}
C_{n}^{y}=C_{n+1}^{y} \quad \mathrm{n}=1,2, \ldots(\mathrm{N}-1) \\
K_{n} \frac{\partial C_{n}^{y}}{\partial z}=K_{n+1} \frac{\partial C_{n+1}^{y}}{\partial z} \quad \mathrm{n}=1,2, \ldots(\mathrm{N}-1)
\end{gathered}
$$

must be considered, in order to be possible to uniquely determine the $2 \mathrm{~N}$ arbitrary constants appearing in the solution of the set of problems (8).

Now, applying the Laplace transform in equation (8) results in:

$$
\begin{aligned}
& \frac{d^{2} \bar{C}_{n}^{y}(s, z, p)}{d z^{2}}-\frac{\left(p+\lambda+s u_{n}-K_{x} s^{2}\right) \bar{C}_{n}^{y}(s, z, p)}{K_{z}}= \\
& \frac{\left(K_{x} s-u_{n}\right) \bar{C}_{n}^{y}(0, z, p)}{K_{z}}
\end{aligned}
$$

with the initial condition:

$$
\bar{C}^{y}(s, z, 0)=0 \quad \text { at } \mathrm{t}=0
$$

source condition:

$$
\bar{C}^{y}(0, z, p)=\frac{Q}{u}\left(\frac{1}{s}-\frac{e^{-p t_{r}}}{s}\right) \delta\left(z-H_{s}\right) \quad \text { at } \quad \underset{(12)}{\mathrm{x}}=0
$$

and the boundary conditions:

$$
K_{z} \frac{d \bar{C}^{y}(s, z, p)}{d z}=0 \quad \text { at } \quad z=h
$$


and

$$
K_{z} \frac{d \bar{C}^{y}(s, z, p)}{d z}=0 \quad \text { at } \quad z=0
$$

where $\bar{C}^{y}(s, z, p)=L\left\{C^{y}(x, z, t) ; x \rightarrow s ; t \rightarrow p\right\}$, which has the solution:

$$
\begin{aligned}
& \bar{C}_{n}^{y}(s, z, p)=A_{n} e^{-R_{n} z}+B_{n} e^{R_{n} z}+\frac{\left(1-e^{-p t_{r}}\right)}{p} \frac{Q}{R_{a}} \\
& \left(e^{-R_{n}\left(z-H_{s}\right)}-e^{R_{n}\left(z-H_{s}\right)}\right)
\end{aligned}
$$

where

$$
\begin{aligned}
& R_{n}=\sqrt{\frac{1}{K_{z}}\left[p+\lambda+s u_{n}\left(1-\frac{K_{x} s}{u_{n}}\right)\right]} ; \\
& R_{a}=2 \frac{\sqrt{K_{z}\left[p+\lambda+s u_{n}\left(1-\frac{K_{x} s}{u_{n}}\right)\right]}}{\left(1-\frac{K_{x} s}{u_{n}}\right)}
\end{aligned}
$$

Finally, applying the initial and boundary conditions one obtains a linear system for the integration constants. Then the concentration is obtained by inverting numerically the transformed concentration $\bar{C}$ by a Gaussian quadrature scheme (Stroud and Secrest, 1966):

$$
\begin{aligned}
& \text { for } t>t_{r}: \\
& C_{n}^{y}(x, z, t)=\sum_{i=1}^{k} a_{i}\left(\frac{p_{i}}{t}\right) \sum_{j=1}^{m} a_{j}\left(\frac{p_{j}}{x}\right)\left[A_{n} e^{-G_{n} z}+B_{n} e^{G_{n} z}\right. \\
& \left.+Q \frac{\left(1-e^{-p_{i} \frac{t_{r}}{t}}\right)}{F_{n}}\left(e^{-\left(z-H_{s}\right) G_{n}}-e^{\left(z-H_{s}\right) G_{n}}\right) \eta\left(z-H_{s}\right)\right]
\end{aligned}
$$

for $t_{r}>t$ :

$$
\begin{aligned}
& C_{n}^{y}(x, z, t)=\sum_{i=1}^{k} a_{i}\left(\frac{p_{i}}{t}\right) \sum_{j=1}^{m} a_{j}\left(\frac{p_{j}}{x}\right)\left[A_{n} e^{-G_{n} z}+B_{n} e^{G_{n} z}\right. \\
& \left.+\frac{Q}{F_{n}}\left(e^{-\left(z-H_{s}\right) G_{n}}-e^{\left(z-H_{s}\right) G_{n}}\right) \eta\left(z-H_{s}\right)\right]
\end{aligned}
$$

where

$$
G_{n}=\sqrt{\frac{1}{K_{r}}\left[\frac{p_{i}}{t}+\lambda+\frac{p_{j}}{x} u_{n}\left(1-\frac{p_{j}}{P_{o}}\right)\right]}
$$

$$
F_{n}=2 \frac{p_{i}}{t} \frac{\sqrt{K_{z}\left[\frac{p_{i}}{t}+\lambda+\frac{p_{j}}{x} u_{n}\left(1-\frac{p_{j}}{P_{e}}\right)\right]}}{\left(1-\frac{p_{j}}{P_{e}}\right)}
$$

where $\eta$ is the Heaviside function and $P_{e}=u_{n} x / K_{x}$ is the well known Peclet number, essentially representing the ratio between the advective transport to diffusive transport. This can be physically interpreted as the parameter whose magnitude indicates the atmospheric conditions in terms of the strength of winds. Small values of this number may be related to the weak winds when the downwind diffusion becomes important and the region of interest remains close to the source, whereas large values imply moderate to strong winds when the downwind diffusion is neglected in comparison to the advection and the region of interest extends to a larger distance from the source.

The solution is valid for $x>0$ and $t>0$, as the quadrature scheme of Laplace inversion does not work for $x=$ 0 and $t=0$. The constants $a_{i}, a_{j}$, and $p_{i}, p_{j}$ are the weights and roots of the Gaussian quadrature scheme and are tabulated in the book by Stroud and Secrest (1966) while $\mathrm{k}$ and $\mathrm{m}$ are the quadrature points. However, we are aware of the existence in the literature of more accurate methods to evaluate this integral, like the multi-precision approach (Abate and Valkó, 2004). The semi-analytical character of the solutions (14) and (15) reduces to the solution of the Moreira et al. (1999) when the time goes to infinity $(t \rightarrow \infty), P_{e} \rightarrow \infty$ and $\lambda=0$.

In order to show pollutant numerical simulations for a time-dependent three-dimensional, we finalize reporting a simplified solution for this sort of problem, reliable for some physical scenarios. Indeed, we assume that the time-dependent three-dimensional solution is written in terms of the time-dependent two-dimensional solution multiplied by the steady Gaussian function in the $y$-direction. This procedure yields:

$$
C(x, y, z, t)=C^{y}(x, z, t) \frac{e^{\left(-y^{2} / 2 \sigma_{y}^{2}\right)}}{\sqrt{2 \pi} \sigma_{y}}
$$

where $C^{y}(x, z, t)$ is expressed by the previous discussed formulation. 


\section{Boundary layer parameterization}

In the atmospheric diffusion problems the choice of a turbulent parameterization represents a fundamental aspect for pollutant dispersion modeling. The reliability of each model strongly depends on the way the turbulent parameters are calculated and is related to the current understanding of the ABL (Mangia et al., 2002). In order to calculate the three-dimensional concentration in the ground-level centerline concentration (Eq. 14) we need to know the lateral dispersion parameter . For a Convective Boundary Layer (CBL) (Copenhagen experiment), we used the lateral dispersion parameter $\sigma_{y}$ derived by Degrazia et al. (1998). It presents the following form:

$$
\frac{\sigma_{y}^{2}}{z_{i}^{2}}=\frac{0.21}{\pi} \int_{0}^{\infty} \sin ^{2}\left(2.26 \psi^{1 / 3} X n^{\prime}\right) \frac{d n^{\prime}}{\left(1+n^{\prime}\right)^{5 / 3} n^{\prime 2}}
$$

where $X$ is a non-dimensional distance $\left(X=x w_{*} / u h\right)$, is the convective velocity scale and $h$ is the top of the CBL.

The equation (18) contain the unknown function $\psi$, the molecular dissipation of turbulent velocity is a leading destruction terms in equations for the budget of second-order moments, and according Hфjstrup (1982), has the form:

$$
\psi^{1 / 3}=\left[\left(1-\frac{z}{h}\right)^{2}\left(\frac{z}{-L}\right)^{-2 / 3}+0.75\right]^{1 / 2}
$$

where $L$ is the length of Monin-Obukhov defined in the surface boundary layer.

In terms of the convective scaling parameters the vertical eddy diffusivity can be formulated as (Degrazia et al., 1997):

$$
\begin{aligned}
& \frac{K_{z}}{w_{*} h}=0.22\left(\frac{z}{h}\right)^{1 / 3}\left(1-\frac{z}{h}\right)^{1 / 3}\left[1-\exp \left(-\frac{4 z}{h}\right)\right. \\
& \left.-0.0003 \exp \left(\frac{8 z}{h}\right)\right]
\end{aligned}
$$

The equations used by the model to calculate mean wind are those predicted by similarity theory (Panofsky and Dutton, 1988):

$$
u=\frac{u_{*}}{k}\left[\ln \frac{z}{z_{0}}-\psi_{m}\left(\frac{z}{L}\right)\right]
$$

where $u_{*}$ is the scale velocity relative to mechanical turbulence, $k$ the von Karman constant, $z_{0}$ is the roughness length and $\Psi$ the stability function expressed in Businger relations:

$$
\psi_{m}\left(\frac{z}{L}\right)=\ln \left(\frac{1+x^{2}}{2}\right)+\ln \left(\frac{1+x}{2}\right)^{2}-2 \arctan x+\frac{\pi}{2}
$$

for $1 / L<0$

$$
\text { with } x=(1-15 z / L)^{1 / 4} \text {. }
$$

\section{Numerical simulations}

The solution (15) and (16) are dependent on $t_{r}$ and $\lambda$ parameters. Then, the sensitivity of the ground-level concentration to each parameter is tested. Firstly, to show an example of the application of the obtained solution (16) $\left(t_{r}>t\right)$, we report in Figure 1 the time evolution of non-dimensional concentration $C^{y} u h / Q$ at three downwind distances ( $x=500,1000$ and 2000m).

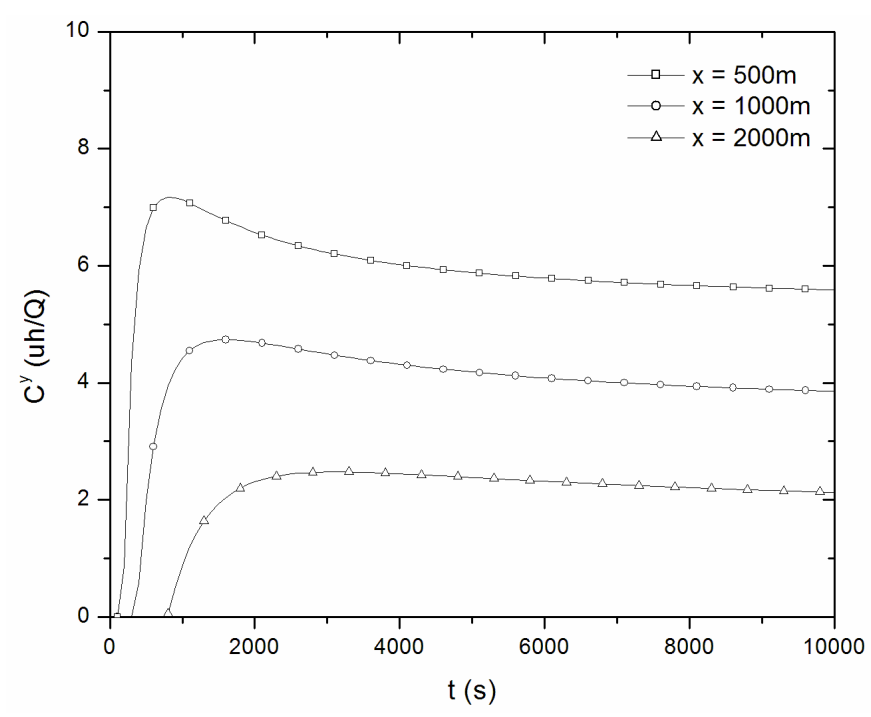

Figure 1: Time evolution of non-dimensional concentration at three downwind distances $(x=500 \mathrm{~m}$, 1000 and 2000).

Figure 2 shows the non-dimensional ground level concentration ( $\left.C^{y} u h / Q\right)$ as a function of the source distance with variable duration releases $\left(t_{r}=50,100\right.$, $150,200 \mathrm{~s})$ for three different times ( $t=250,500$ and 750s) emitted through a stack with a physical height of $10 \mathrm{~m}$, in micrometeorological conditions characterized by a $2 \mathrm{~m} / \mathrm{s}$ wind velocity, a $1100 \mathrm{~m}$ mixing layer, $\mathrm{w}^{*}=2 \mathrm{~m} / \mathrm{s}$ and $\mathrm{L}=-10 \mathrm{~m}$.

Figure 2 shows that the concentration peak values increases as the duration of the release grows longer, until it reaches a limit value, for sufficiently long durations of the release. Besides, the concentration peak 
values decrease with the increase of the source distance.

Figure 3 shows the concentration time evolutions with the parameters of the reference situation for release duration of 50 s for different values of the chemical-phy-
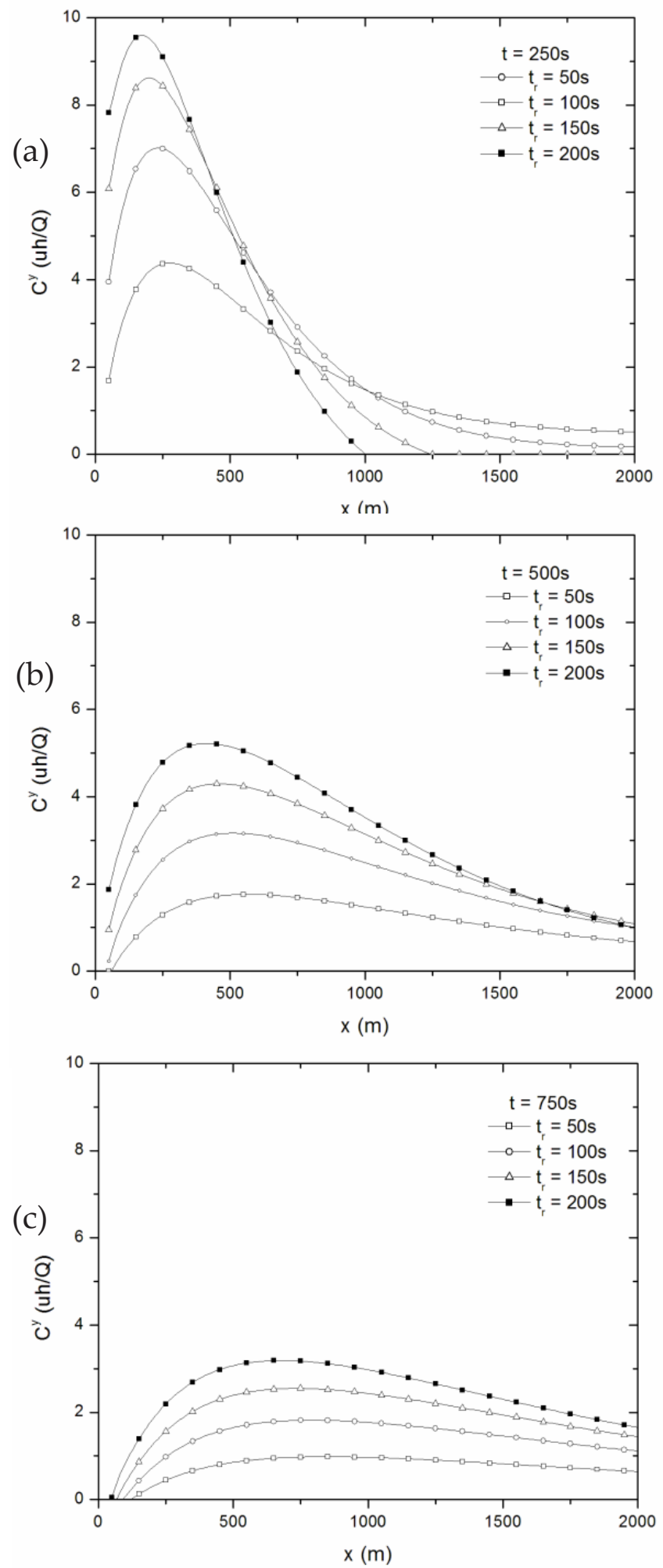

Figure 2: Crosswind integrated concentration as a function of the source distance for three different times ( $t=250,500$ and 750s) and different durations of release $\left(t_{r}=50,100,150\right.$ and $\left.200 s\right)$. sical decay coefficient $(\lambda)$ at downwind distance of 500 and $1000 \mathrm{~m}$.

Figure 3 show the time evolution of concentration at downwind distance of 500 and $1000 \mathrm{~m}$ for time release of 50s and different chemical-physical decay constants. Figure 3 shows that the concentration peak values decrease as the downwind distance increase. Also, as the downwind distance increase, the peak concentration variation between the chemical-physical decay constants, increase.
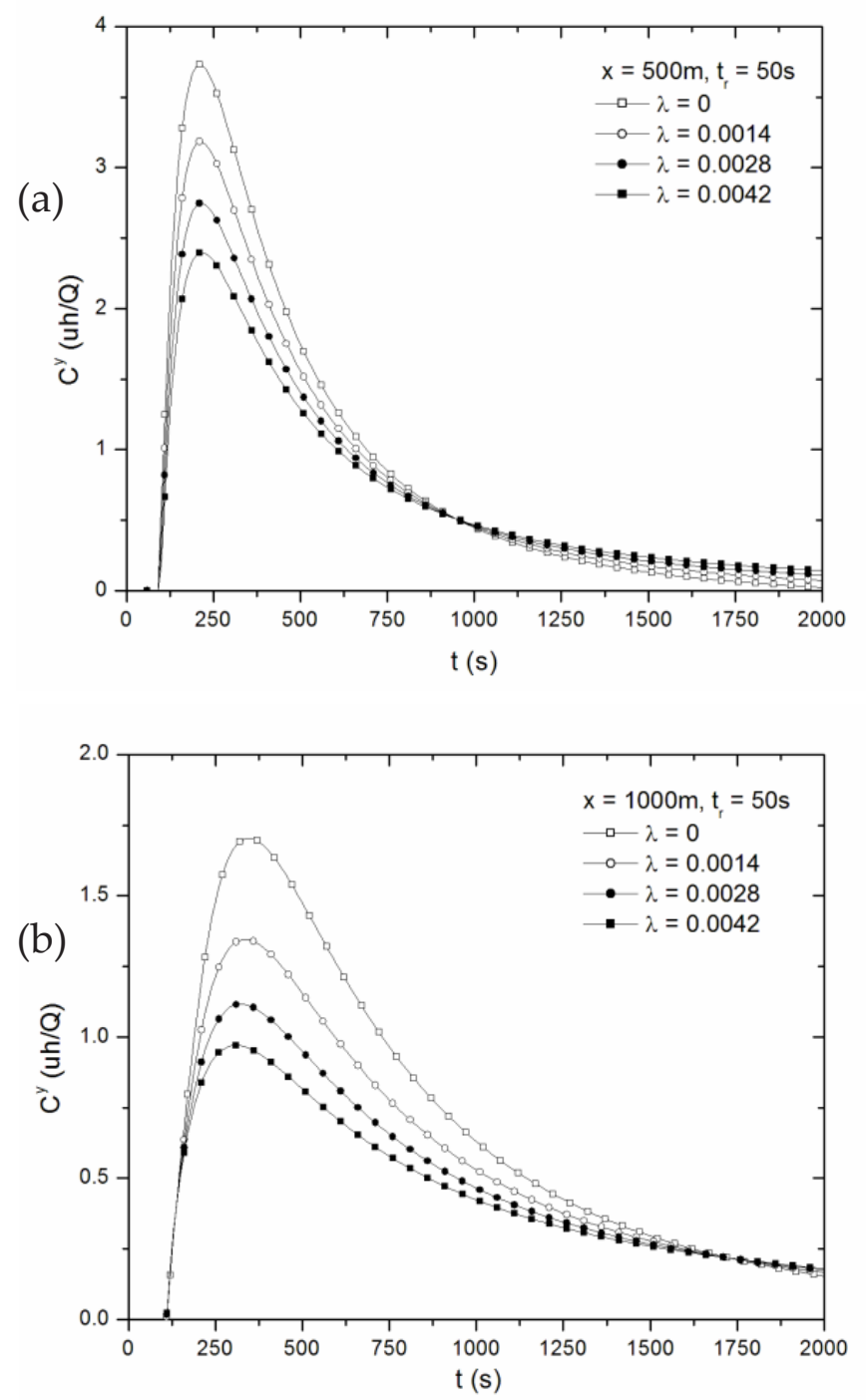

Figure 3: Time evolution of concentration at downwind distance of $500 \mathrm{~m}$ and $1000 \mathrm{~m}$ for time release of 50s and different chemical-physical decay constants.

Figure 4 shows concentration distributions in the horizontal $x y$-plane at ground-level for six different times: $t=100,500,100,2000,3000$ and 5000s. These isolines of equal concentration corresponds the solution $t_{r}>t$.

As expected, the time becomes longer, the concentrations values enter into a steady-state condition. 


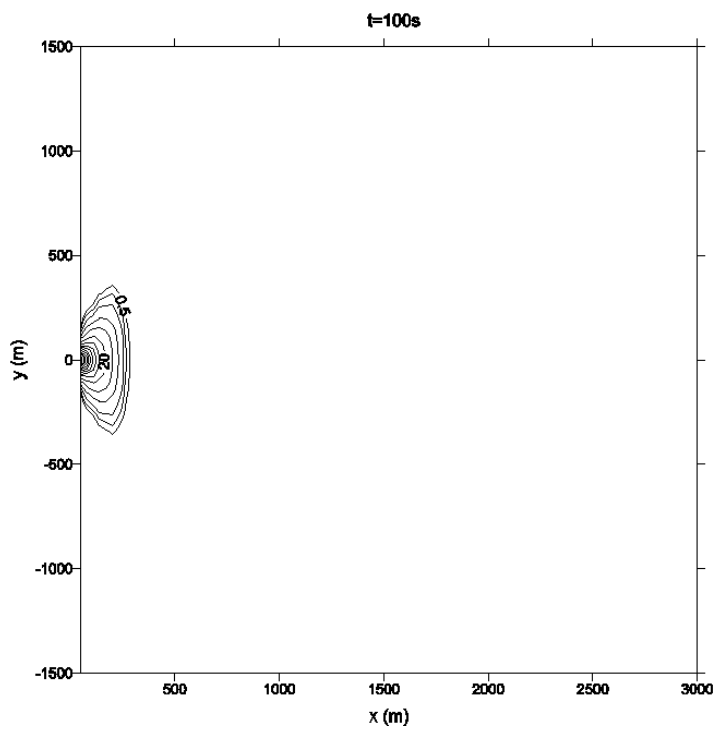

(a)

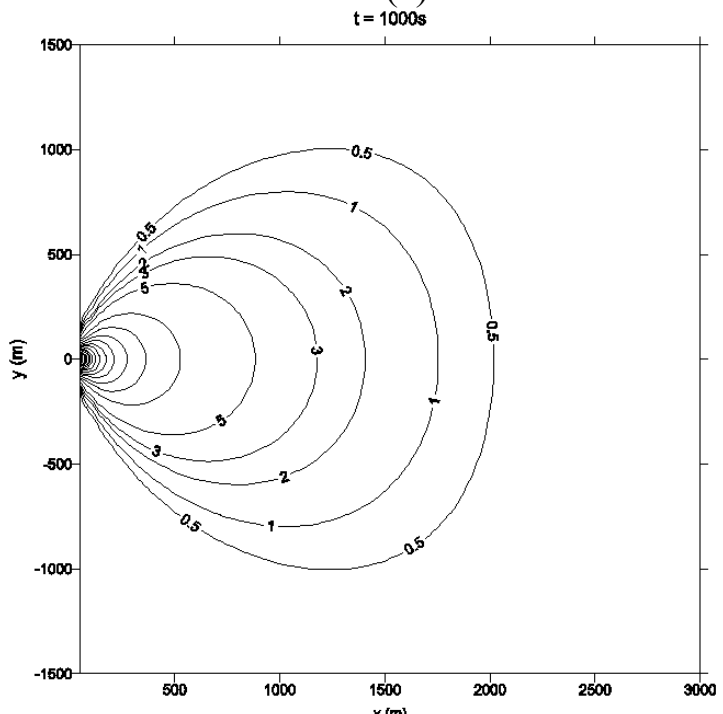

(c)

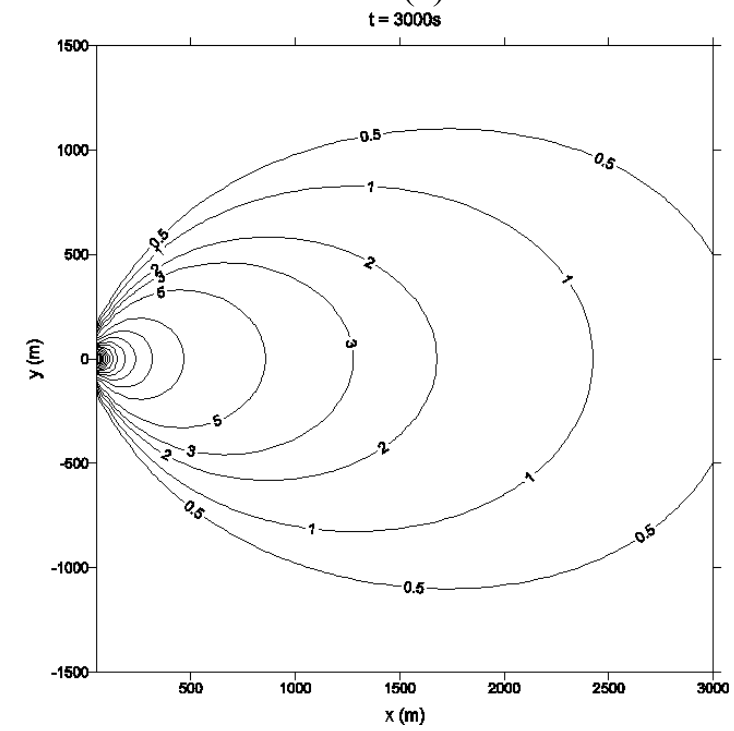

(e)

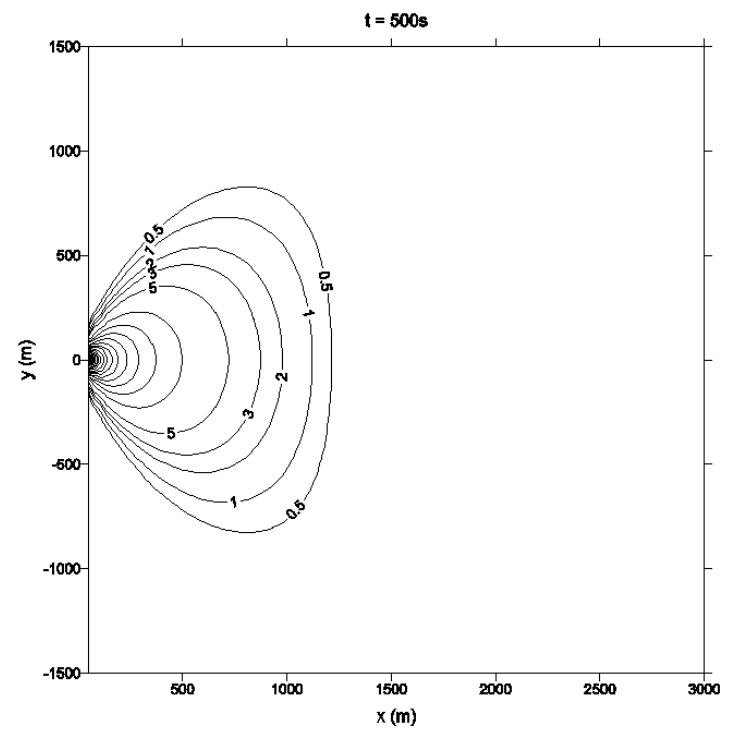

(b)

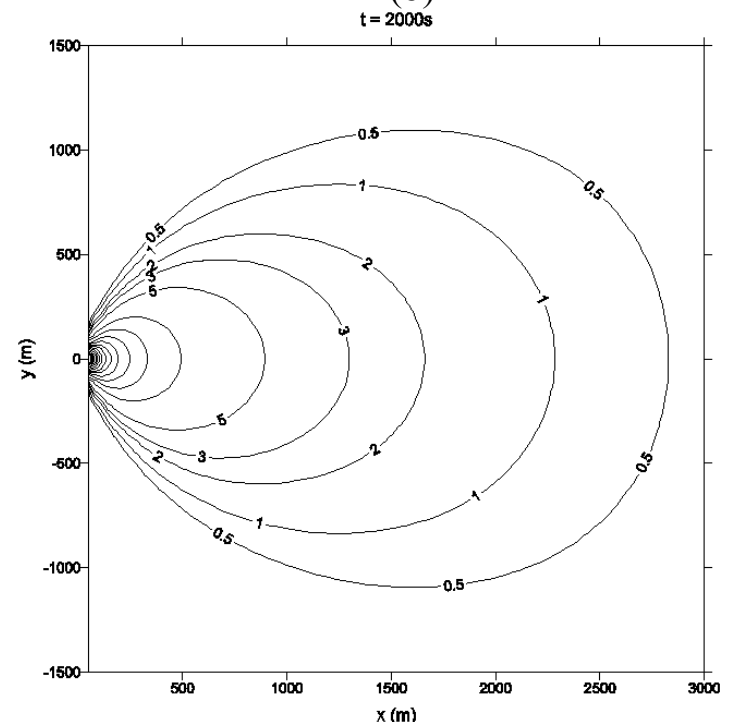

(d)

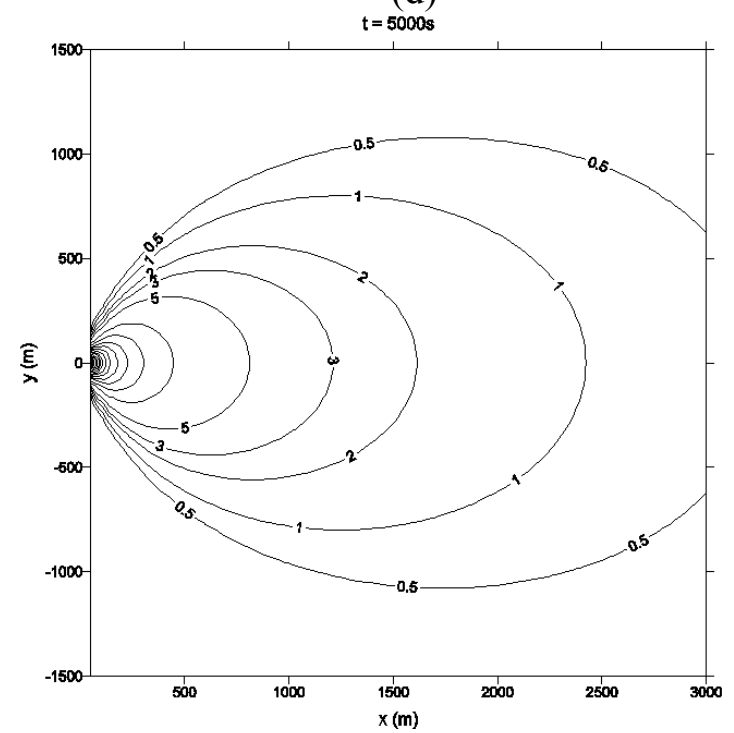

(f)

Figure 4: Concentrations distributions in the horizontal $\mathrm{xy}$-plane at ground-level for six different times: $\mathrm{t}=$ $100,500,100,2000,3000$ and 5000s, for the solution . 
Figure 5 shows concentration distributions in the horizontal $x y$-plane at ground-level for the time $t=1000 \mathrm{~s}$ and four different time release $\left(t_{r}=300,500,800\right.$ and $950 \mathrm{~s})$. The lines represent isolines of equal concentration.

As the duration of the release becomes longer, the concentrations values enter into a steady-state condition. Comparing the Figures 4 for $t=1000$ s and Figures 5 this affirmative is clearly observed.

Furthermore, we evaluated the performance of the model with the boundary layer parameterization pro-

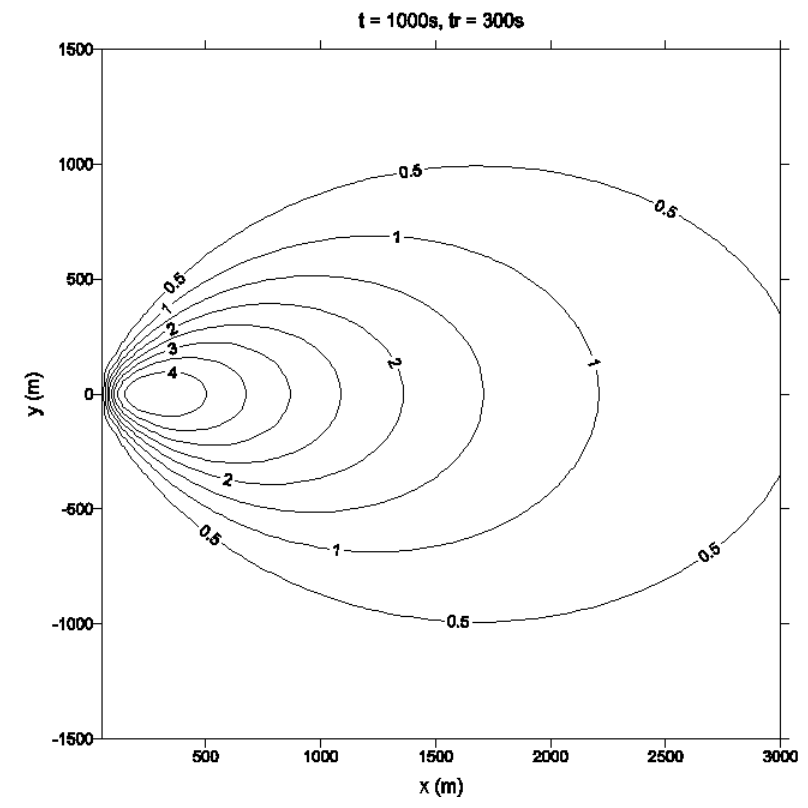

(a)

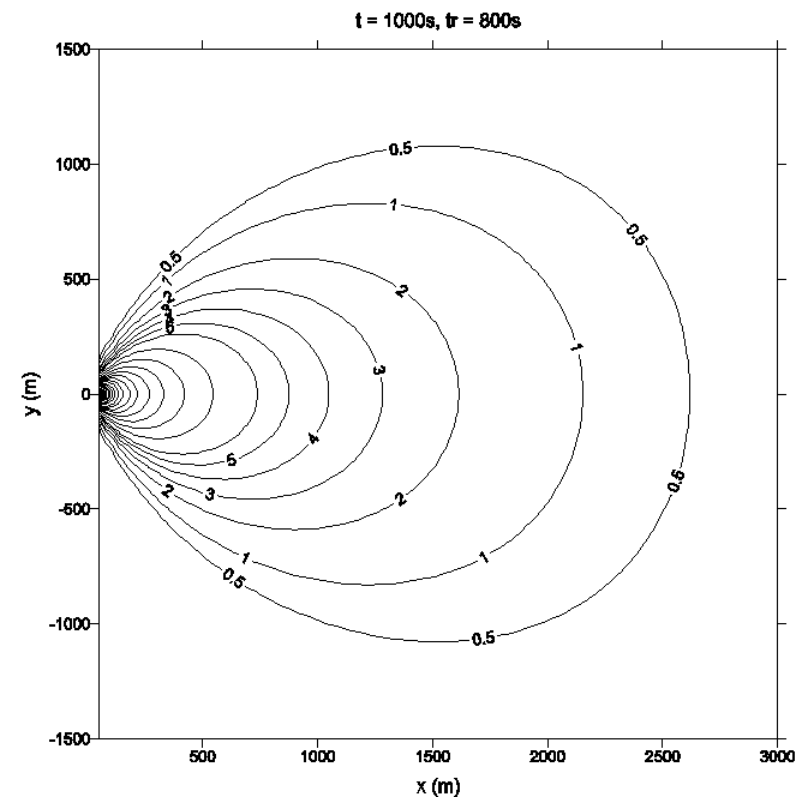

(c) posed, using the Copenhagen data set (Gryning and Lyck, 1984). The Copenhagen data set is composed of tracer SF6 data from dispersion experiments carried out in northern Copenhagen. The tracer was released without buoyancy from a tower at a height of $115 \mathrm{~m}$ and was collected at ground-level positions in up to three crosswind arcs of tracer sampling units. The sampling units were positioned 2-6 $\mathrm{km}$ far from the point of release. We used the values of the crosswind integrated concentrations normalized with the tracer release rate

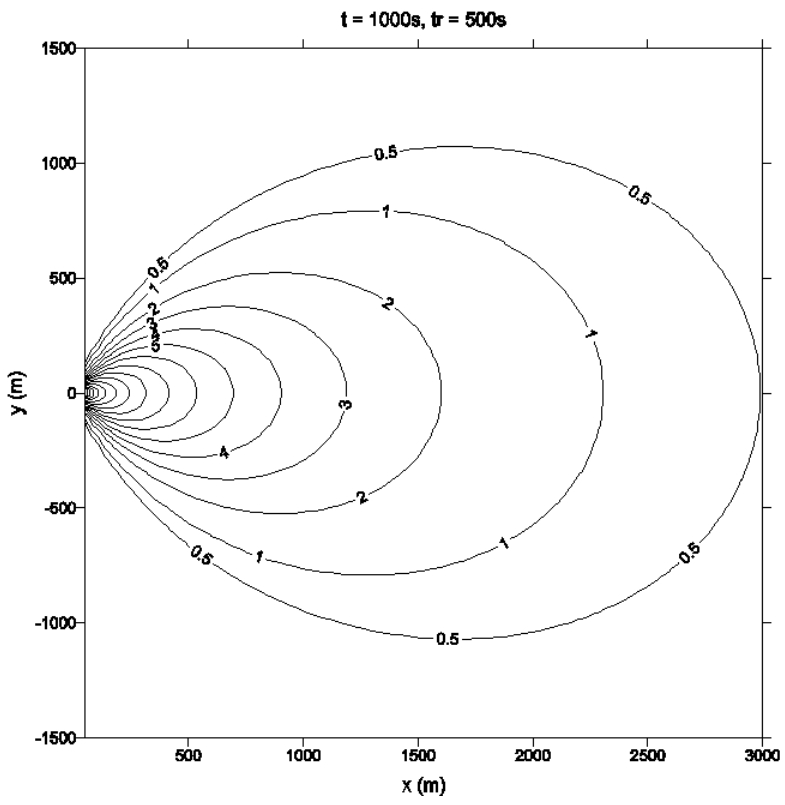

(b)

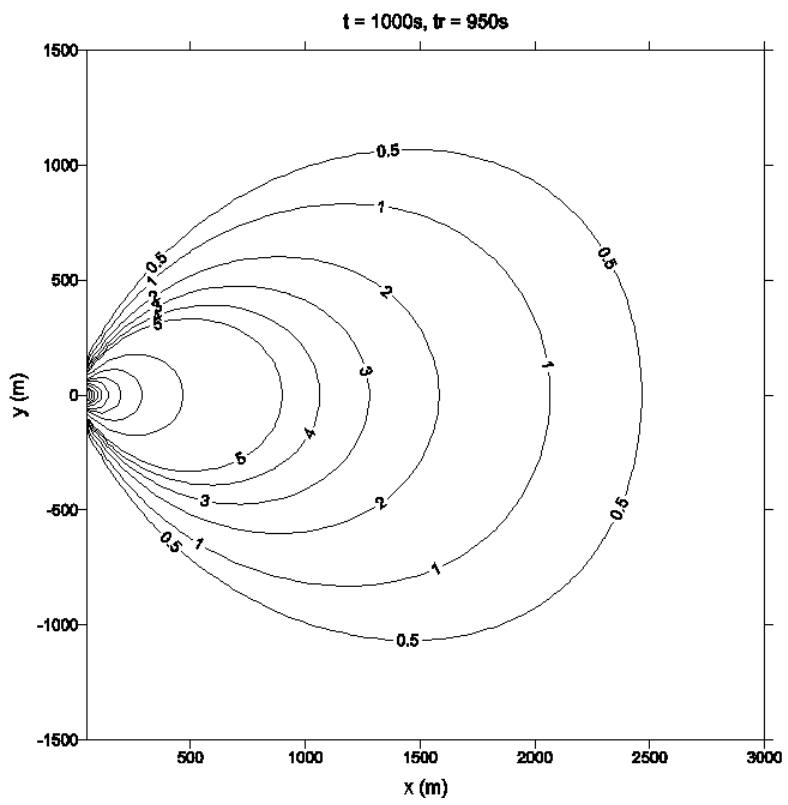

(d)

Figure 5: Concentrations distributions in the horizontal $x y$-plane at ground-level for $t=1000$ s and four different times releases: $\mathrm{t}_{\mathrm{r}}=300,500,800$ and $950 \mathrm{~s}$, for the solution . 
from Gryning et al. (1987). Tracer releases typically started up 1 hour before the tracer sampling and stopped at the end of the sampling period. The site was mainly residential with a roughness length of $0.6 \mathrm{~m}$. Generally the distributed data set contains hourly mean values of concentrations and meteorological data. However, in this work, we used data with a greater time resolution. In particular, we used 20 minutes averaged measured concentrations and 10 minutes averaged values for meteorological data. In such manner, in this work, the variables $\left(L, u^{*}, w^{*}\right)$ in the Copenhagen data set are dynamical variables (except the variable $h$ ). For details of the experimental data see the works of Tirabassi and Rizza (1997) and Costa et al. (2006).

The results obtained with the ADMM method are compared with the M4PUFF model (Tirabassi and Rizza, 1997) which is based on a general technique for solving the K-equation using the truncated Gram-Charlier expansion (type A) of the concentration field and a finite set equation for the corresponding moments. Table 1 presents some performance measurements, obtained using the well-known statistical evaluation procedure described by Hanna (1989). The statistical index FB indicates whether the predicted quantities underestimate or overestimate the observed ones. The statistical index NMSE represents the quadratic error of the predicted quantities in relation to the observed ones. The best results are indicated by values nearest 0 in NMSE, FB, and FS, and nearest 1 in COR and FA2.

Table 1. Statistical evaluation of model results (Copenhagen dataset).

\begin{tabular}{cccccc}
\hline \hline Model & NMSE & R & FA2 & FB & FS \\
\hline ADMM Unstable & 0.15 & 0.81 & 0.95 & 0.18 & 0.38 \\
M4PUFF Unstable & 0.21 & 0.74 & 0.90 & 0.10 & 0.45 \\
\hline
\end{tabular}

The statistical indices point out that a good agreement is obtained between experimental data and the ADMM model. A more detailed inspection of the Table 1 permits to stress that the ADMM simulate well the observed concentrations presenting the best values for NMSE, COR (81\%) and FA2 (95\%).

\section{Conclusions}

A solution of the unsteady-state two-dimensional advection-diffusion equation has been presented that considers the duration time release and chemical-physical decay and can be applied for describing the turbulent dispersion of many scalar quantities, such as air pollution, radioactive material, heat, and so on. From the previous results, we promptly notice the aptness this model to understand the time evolution of the concentration and its dependency on the duration of the contaminant emission. In fact, this model, allow us to simulate the continuous, short-term and instantaneous emissions. In particular, the model is suitable for an initial and rapid assessment of atmospheric dispersion under emergency conditions without sophisticated computing resources.

To show the performances of the solution in actual scenarios, a parameterization of the ABL has been introduced, and the values predicted by the solutions have been compared with experiment dataset. The analysis of the results shows a reasonably good agreement between the computed values and the experimental ones. The discrepancies with the experimental data depend not on the solution of the advection-diffusion equation but on the equation itself, which is only a model of the reality. Moreover, a source of discrepancies between the predicted and measured values lies in the ABL parameterization used (i.e., vertical wind and eddy diffusivity profiles). Although models are sophisticated instruments that ultimately reflect the current state of knowledge on turbulent transport in the atmosphere, the results they provide are subject to a considerable margin of error. This is due to various factors, including in particular the uncertainty of the intrinsic variability of the atmosphere. Models, in fact, provide values expressed as an average, that is, a mean value obtained by the repeated performance of many experiments, whereas the measured concentrations are a single value of the sample to which the ensemble average provided by models refers. This is a general characteristic of the theory of atmospheric turbulence and is a consequence of the statistical approach used in attempting to parameterize the chaotic character of the measured data. In light of the above considerations, an analytical solution is useful for evaluating the performances of sophisticated numerical dispersion models (which numerically solve the advection-diffusion equation), yielding results that can be compared not only with experimental data but, in an easy way, with the solution itself, to check numerical errors without the uncertainties presented above.

\section{Ackowledgements}

The author thank CNPq for the partial financial support of this work.

\section{References}

Arya, S.P., 1999. Air pollution meteorology and dispersion. Oxford University Press, New York, 320 pp.

Abate, J. and Valkó, P.P., 2004. Multi-precision Laplace transform inversion. International Journal for Numerical Methods in Engineering 60, 979-993. 
Bianconi, R. and Tamponi, M., 1993. A mathematical model of diffusion from a steady source of short duration in a finite mixing layer. Atmos. Environ. 27A, 5, 781-792.

Brebbia, C.A.,1981. Progress in boundary element methods. Pentech press London, 1, 315pp.

Chock, D.P., Sun, P. and Winkler, S.L., 1996. Trajectory-grid: an accurate sign-preserving advection-diffusion approach for air quality modeling. Atmos. Environ. 30(6), 857-868.

Costa, C.P., Vilhena, M.T., Moreira, D.M. and Tirabassi, T., 2006. Semi-analytical solution of the steady three-dimensional advection-diffusion equation in the planetary boundary layer. Atmos. Environ. 40(29), 5659-5669.

Degrazia, G.A., Campos Velho, H.F. and Carvalho, J.C., 1997. Nonlocal exchange coefficients for the convective boundary layer derived from spectral properties. Contr. Atmos. Phys., 57-64.

Degrazia, G.A., Mangia, C. and Rizza U., 1998. A comparison between different methods to estimate the lateral dispersion parameter under convective conditions. Journal of Applied Meteorology 37, 227-231.

Gryning, S.E., Lyck, E. 1984. Atmospheric dispersion from elevated source in an urban area: comparison between tracer experiments and model calculations. Journal of climate Applied Meteorology 23, 651654.

Gryning, S.E., Holtslag, A.A.M., Irwin, J.S. and Siversten, B., 1987. Applied dispersion modelling based on meteorological scaling parameters. Atmos. Environ. 21, 7989.

Hanna, S.R., 1989. Confidence limit for air quality models as estimated by bootstrap and jacknife resampling methods. Atmos. Environ. 23, 13851395.

Højstrup, J., 1982. Velocity spectra in the unstable boundary layer. J. Atmos. Sci. 39, 2239-2248.

Huang, C.H., 1979. A theory of dispersion in turbulent shear flow. Atmos. Environ. 13, 453-461.

Huebner, K.H.H., Dewhirst, D.L., Smith, D.E., Byrom, T.G., 2001. Finite element method for engineers. J. Wiley \& Sons, New York, 744pp.

Lin, J.S. and Hildemann, L.M., 1997. A generalised mathematical scheme to analytically solve the atmospheric diffusion equation with dry deposition. Atmos. Environ. 31, 59-71.

Mangia, C., Moreira, D.M., Schipa, I., Degrazia, G.A., Tirabassi, T. and Rizza, U., 2002. Evaluation of a new eddy diffusivity parameterisation from turbulent Eulerian spectra in different stability conditions. Atmos. Environ. 36, 67-76.

Moreira, D.M., Degrazia, G.A. and Vilhena, M.T., 1999. Dispersion from low sources in a convective boundary layer: an analytical model. Il Nuovo Cimento 22C, n.5, 685-691.

Moreira, D.M., Santos, D.P., Carvalho, J.C., Vilhena, M.T., Degrazia, G.A. and Tirabassi, T., 2004. A solution of the steady K-diffusion equation for ABL dispersion in inhomogeneous turbulence with plume rise. Revista Brasileira de Meteorologia 19, 2, 113-122.

Moreira, D.M, Vilhena, M.T., Tirabassi, T., Costa, C. and Bodmann, B., 2006. Simulation of pollutant dispersion in the atmosphere by the Laplace transform: the ADMM approach. Water, Air and Soil Pollution 177, 411-439.

Panofsky, H.A. and Dutton, J.A., 1988. Atmospheric Turbulence. John Wiley \& Sons, New York.

Pasquill, F., and Smith, F. B., 1983. Atmospheric diffusion. John Wiley \& Sons, New York.

Rizza, U., Gioia, G., Mangia, C. and Marra, G.P., 2003. Development of a grid dispersion model in a large eddy simulation generated planetary boundary layer. Il Nuovo Cimento 26C, n.3, 297-309.

Seinfeld, J.H. and Pandis, S.N., 1998. Atmospheric chemistry and physics. John Wiley \& Sons, New York.

Sharan, M. Kansa, E.J. and Gupta, S., 1997. Application of the multiquadric method for numerical solution of elliptic partial differential equations. Applied Mathematics and Computation 84, 275-302.

Sharan, M., Modani, M., and Yadav, A.K., 2003. Atmospheric dispersion: an overview of mathematical modeling framework. Proc. Indian National. Sci. Acad. 69A, 725-744.

Stroud, A.H. and Don Secrest, 1966. Gaussian Quadrature Formulas, Englewood Cliffs, N.J., Prentice Hall, Inc. 
Tangerman, G., 1978. Numerical simulations of air pollutant dispersion in a stratified planetary boundary layer. Atmos. Environm. 12, 1365-1369.

Tirabassi, T., 1989. Analytical air pollution and diffusion models. Water, Air and Soil Pollution 47, 19-24.

Tirabassi, T. and Rizza, U., 1997. Boundary layer parameterization for a non-Gaussian puff model. J. Appl. Meteor. 36, 1031-1037.

Tirabassi, T., 2003. Operational advanced air pollution modeling. PAGEOPH 160 (1-2), 5-16.

Zannetti, P., 1990. Air Pollution Modelling. Computational Mechanics Publications, Southampton, 444pp. 